\title{
Metformin mediates resensitivity to 5-fluorouracil in hepatocellular carcinoma via the suppression of YAP
}

\author{
Yu Tian ${ }^{1}$, Bo Tang ${ }^{2}$, Chengye Wang ${ }^{1}$, Deguang Sun ${ }^{1}$, Rixin Zhang ${ }^{1}$, Nan Luo ${ }^{3}$, Zhao \\ Han $^{1}$, Rui Liang ${ }^{1}$, Zhenming Gao ${ }^{1}$, Liming Wang ${ }^{1}$ \\ ${ }^{1}$ Division of Hepatobiliary and Pancreatic Surgery, Department of Surgery, The Second Affiliated Hospital of Dalian Medical \\ University, Dalian, Liaoning, P.R. China \\ ${ }^{2}$ Department of Hepatobiliary Surgery, Affiliated Hospital of Guilin Medical University, Guilin, Guangxi, P.R. China \\ ${ }^{3}$ Department of Infection, The Second Affiliated Hospital of Dalian Medical University, Dalian, Liaoning, P.R. China \\ Correspondence to: Liming Wang, email: wangbcc259@163.com \\ Bo Tang, email: dytangbo@163.com \\ Keywords: metformin, hepatocellular carcinoma, 5-fluorouracil, YAP
}

Received: March 30, $2016 \quad$ Accepted: May 29, $2016 \quad$ Published: June 15, 2016

\section{ABSTRACT}

Metformin plays an anti-proliferative role in tumor cells in many types of cancer. However, the correlation between metformin and sensitivity to chemotherapeutic agents in hepatocellular carcinoma (HCC) and the relevant mechanism are unclear. The present study showed that HCC patients with type 2 diabetes mellitus benefited from metformin administration, with a longer overall survival. Metformin resensitized Bel-7402/5-fluorouracil (Bel/Fu) cells to 5-fluorouracil (5-Fu) in vitro and in vivo, and the combination of metformin and 5-Fu inhibited cell proliferation, promoted cell apoptosis and induced G0/G1 cell cycle arrest in the Bel/Fu cells. Moreover, metformin repressed YAP by both decreasing the total protein expression and accelerating the phosphorylation of YAP. The inhibition of YAP subsequently promoted the expression of PTEN, and suppressed the Akt pathway. Therefore, the expression of P-gP and MRP1 was downregulated. Taken together, our findings suggested that metformin may increase sensitivity to chemotherapeutic agents by suppressing YAP in hepatocellular carcinoma.

\section{INTRODUCTION}

Hepatocellular carcinoma (HCC) is of one the most aggressive malignancies worldwide. It is the sixth most common cancer on the global scale and the thirdleading cause of cancer-related mortality $[1,2]$. Surgical resection and liver transplantation may offer curative opportunities to select patients. However, the majority of HCC patients are diagnosed at an advanced stage and have lost the opportunity for surgical treatment $[3,4]$. In this case, locoregional therapy, such as transarterial chemoembolization (TACE), is a feasible option, despite its low rate of efficacy and its associated small improvements in survival $[5,6]$. One major drawback of TACE is the development of low sensitivity to chemotherapeutic agents [7]. Therefore, it is urgent to identify novel pharmacological strategies for HCC management.
Recent studies have shown that components of metabolic syndrome, including diabetes mellitus (DM), obesity and hyperlipidemia, are significant risk factors for $\mathrm{HCC}$, and that the presence of DM is highly associated with the development of $\mathrm{HCC}[8,9]$. Metformin is a widely used oral anti-hyperglycemic agent for type 2 diabetes mellitus (T2DM). Systemically, it reduces glycemia by inhibiting hepatic gluconeogenesis and increasing insulin sensitivity in peripheral tissues. Recent epidemiological evidence has indicated that the use of metformin can decrease both the incidence and the mortality of multiple cancers [10], suggesting its potential role as an adjuvant agent for anti-cancer activity. However, the role and the underlying mechanism of metformin's effects on cancer-related chemotherapeutic agents' sensitivity remain unclear.

The Hippo signaling pathway is crucial in regulating tissue growth, stem cell activity and tumorigenesis [11]. 
YAP, first reported in 1994 as a binding partner of the $\mathrm{SH} 3$ domain of the Yes proto-oncogene product [12], is a key downstream effector of the Hippo signaling pathway. When the Hippo pathway is deactivated, YAP can translocate to the nucleus to activate gene expression by associating with a number of DNA-binding transcription factors, modulating diverse cellular functions, including proliferation, apoptosis, migration and differentiation $[13,14]$. YAP is generally overexpressed in tumor tissues [15-17], and has been reported to modulate the epithelialmesenchymal transition (EMT) and various pathways that are important for resistance to chemotherapy [18, 19]. Therefore, we hypothesized that YAP may play an important role in regulating chemotherapeutic sensitivity.

In this study, we showed that patients with $\mathrm{HCC}$ and T2DM who were treated with TACE benefited from metformin administration. Additionally, we presented evidence that metformin increased the sensitivity of $\mathrm{Bel} / \mathrm{Fu}$ cells to $5-\mathrm{Fu}$ both in vitro and in vivo, and that the suppression of YAP may be an important underlying mechanism of this effect.

\section{RESULTS}

\section{Metformin administration resulted in longer OS in HCC patients with T2DM}

To identify whether metformin facilitates resensitization to chemotherapeutic agents in $\mathrm{HCC}$, we examined the curative effect of TACE in HCC patients with T2DM by administering metformin and other antidiabetic agents. The clinical features of the two studied patient cohorts, including age, gender, clinical stage, tumor size, tumor number and serum AFP level expression, are summarized in Table 1. No relationships were observed between the status of metformin administration and the other patient characteristics, including age, gender, serum AFP level, tumor size and tumor number.

The median follow-up period was 35.1 months (ranging from 6 to 78 months) for the HCC patients. The difference in survival rate between the metformin group and the other antidiabetic agents group was evaluated. Kaplan-Meier analysis showed that metformin administration strongly predicted longer OS $(P<0.01$, Figure 1).

\section{Metformin increased sensitivity to chemotherapeutic agents of $\mathrm{HCC}$ in vitro and in vivo}

As metformin administration helps HCC patients with T2DM obtain a better prognosis, we performed an in vitro investigation to test whether metformin could enhance chemotherapeutic sensitivity. Bel/Fu cells treated with metformin exhibited significantly promotion of sensitivity to 5-Fu (Figure 2A). The same effect of metformin could be observed in HepG2 and SKHep-1 cells. Combination with metformin did enhanced efficiency of chemotherapeutic agents, such as 5-Fu, doxorubicin (DOX) and sorafenib (Sor) (Supplementary Figure S1A - S1H). Then, Bel/Fu cells were separately treated with negative control (NC), $100 \mu \mathrm{g} / \mathrm{ml} 5-\mathrm{Fu}(5-$ $\mathrm{Fu}$ ), $10 \mathrm{mM}$ metformin (Met) and a combination of these agents (Com). Cell proliferation was evaluated by CCK8 assay. The course of proliferation was assessed for 3 days after treatment. Met and Com treatments exhibited effective inhibition of cell proliferation compared with the 5-Fu group, especially in the Com group (Figure 2B). We further conducted cell colony formation assays to demonstrate whether metformin combined with $5-\mathrm{Fu}$ inhibited cell proliferation in Bel/Fu cells. The number of colonies formed was $373.0 \pm 9.8$ for the $\mathrm{NC}$ cells, $354.3 \pm 14.7$ for the 5 -Fu group, $136.3 \pm 4.2$ for the Met group and $65.0 \pm 8.9$ for the Com group (Figure $2 \mathrm{C}$ and 2D).

To further examine the role of Met in regulating sensitivity to $5-\mathrm{Fu}$ in vivo, we subcutaneously injected $\mathrm{Bel} / \mathrm{Fu}$ cells into nude mice and treated them with the above agents. As expected, tumors grew more slowly in combined treatment than in single-agent treatment (Figure $2 \mathrm{E}-2 \mathrm{G})$. These results indicated that metformin could resensitize $\mathrm{Bel} / \mathrm{Fu}$ cells to 5-Fu.

\section{Combined treatment with 5-Fu and metformin promoted apoptosis in Bel/Fu cells}

To determine whether metformin-induced increased sensitivity to $5-\mathrm{Fu}$ in $\mathrm{Bel} / \mathrm{Fu}$ cells was accompanied by apoptosis, the percentage of apoptosis was examined using flow cytometry after $48 \mathrm{~h}$ of incubation with the different agents. The percentage of apoptotic cells was significantly increased after combination therapy compared with single-agent therapy and negative control treatment. The percentage of apoptotic cells was $2.5 \pm 0.1 \%$ in the $\mathrm{NC}$ group, $2.3 \pm 0.2 \%$ in the $5-\mathrm{Fu}$ group, $4.5 \pm 0.1 \%$ in the Met group and $9.1 \pm 0.5 \%$ in the Com group (Figure $3 \mathrm{~A}$ and $3 \mathrm{~B})$.

The TUNEL assay is commonly used to detect fragmented DNA in cells that undergo apoptosis. In our study, Bel/Fu cells were pretreated with different agents for $48 \mathrm{~h}$ for TUNEL assay, and the cells were stained by TUNEL and DAPI and analyzed by fluorescence microscopy. As shown in Figure 3C, pretreatment with the combination of 5-Fu and metformin produced more green light than pretreatment with other agents, which suggested that combination treatment increased $\mathrm{Bel} / \mathrm{Fu}$ cell apoptosis.

Subsequently, the up-regulation of the proapoptotic proteins cl-caspase 3, cl-PARP and Bax and the down-regulation of the apoptosis suppressor Bcl-2 were observed in the combination treatment group compared with the other groups (Figure 3D). 
Table 1: Association of metformin administration with patient's characteristics in hepatocellular carcinoma

\begin{tabular}{|c|c|c|c|c|}
\hline Variables & Metformin & No Metformin & Total & $P^{a}$ \\
\hline Age (y) & & & & 0.056 \\
\hline$\leq 50$ & $6(35.3 \%)$ & $11(64.7 \%)$ & 17 & \\
\hline$>50$ & $27(58.7 \%)$ & $19(41.3 \%)$ & 46 & \\
\hline Sex & & & & 0.847 \\
\hline Male & $27(51.9 \%)$ & $25(48.1 \%)$ & 52 & \\
\hline Female & $6(54.5 \%)$ & $5(45.5 \%)$ & 11 & \\
\hline Tumor size $(\mathrm{cm})$ & & & & 0.425 \\
\hline$\leq 5$ & $24(54.5 \%)$ & $20(45.5 \%)$ & 44 & \\
\hline$>5$ & $9(47.4 \%)$ & $10(52.6 \%)$ & 19 & \\
\hline $\operatorname{AFP}(n g / m l)$ & & & & 0.961 \\
\hline$\leq 20$ & $20(52.6 \%)$ & $18(47.4 \%)$ & 38 & \\
\hline$>20$ & $13(52 \%)$ & $12(48 \%)$ & 25 & \\
\hline Tumor number & & & & 0.217 \\
\hline Solitary & $30(55.6 \%)$ & $24(44.4 \%)$ & 54 & \\
\hline Multiple & $3(33.3 \%)$ & $6(66.7 \%)$ & 9 & \\
\hline
\end{tabular}

\section{Combined treatment with 5-Fu and metformin arrested the cell cycle at G0/G1 in Bel/Fu cells}

To explore whether metformin could resensitize $\mathrm{Bel} / \mathrm{Fu}$ cells to 5 -Fu by modifying the cell cycle, we examined cell cycle progression by flow cytometry. $5-\mathrm{Fu}$ combined with metformin arrested the cell cycle at $\mathrm{G} 0 / \mathrm{G} 1$ in $\mathrm{Bel} / \mathrm{Fu}$ cells compared with the other groups (Figure $3 \mathrm{E}$ and $3 \mathrm{~F}$ ). $24 \mathrm{~h}$ after treatment with the different agents mentioned above, the percentage of cells in the G0/G1 phase was $33.4 \pm 0.9 \%$ for the NC group, $35.8 \pm 1.8 \%$ for the 5-Fu group, $40.4 \pm 1.0 \%$ for the Met group and $48.6 \pm$ $1.7 \%$ for the Com group.

Dysfunction of cell cycle checkpoint proteins can alter the progression of the cell cycle. We also examined the G1/S phase proteins cyclin D1, CDK4 and p21. Cyclin D1 and CDK4, which are responsible for the transition from $\mathrm{G} 0 / \mathrm{G} 1$ phase to $\mathrm{S}$ phase, were downregulated following combined treatment. Additionally, p21, a cyclindependent kinase (CDK) inhibitor, was obviously induced following combined treatment (Figure 3G). Thus, these results demonstrated that the combined treatment group arrested Bel/Fu cells in the G0/G1 phase.

\section{Silencing YAP increased sensitivity to 5-Fu in $\mathrm{Bel} / \mathrm{Fu}$ cells}

It has been reported that YAP, a protein abundant with phosphorylation sites (Figure 4A), plays an important role in cancer cell activity, especially in tumor-associated chemoresistance [20]. To investigate the function of YAP in regulating sensitivity to $5-\mathrm{Fu}$ in $\mathrm{HCC}$, we evaluated the expression of YAP in Bel/Fu and Bel cells. As shown in Figure 4B, the YAP protein was highly expressed in Bel/ Fu cells.

To determine whether knockdown of YAP could increase the sensitivity of $\mathrm{Bel} / \mathrm{Fu}$ cells to 5 -Fu, YAP siRNAs were used to silence the expression of YAP. YAP siRNA\#2 repressed YAP protein expression more effectively than other YAP siRNAs (Figure 4C), and the $\mathrm{Bel} / \mathrm{Fu}$ cells transfected with YAP siRNA\#2 displayed a lower proliferative capacity (Figure 4D), a decreased number of colonies (Figure 4E and 4F), an increased cell apoptotic rate (Figure $4 \mathrm{G}$ and $4 \mathrm{H}$ ) and a larger number of cells arrested in the G0/G1 phase (Figure $4 \mathrm{~J}$ and $4 \mathrm{~K}$ ) under $100 \mu \mathrm{g} / \mathrm{ml} \mathrm{5-Fu} \mathrm{than} \mathrm{the} \mathrm{control} \mathrm{siRNA-transfected}$ $\mathrm{Bel} / \mathrm{Fu}$ cells.

Furthermore, the activation of the pro-apoptotic cl-caspase3, cl-PARP and Bax and the downregulation of the apoptosis suppressor Bcl-2 were associated with YAP silencing-induced apoptosis (Figure 4I). Cyclin D1 and CDK4, which are responsible for the transition from the G0/G1 phase to the S phase, were downregulated following YAP knockdown (Figure 4L).

These results indicated that the knockdown of YAP increased sensitivity to $5-\mathrm{Fu}$ in $\mathrm{Bel} / \mathrm{Fu}$ cells. 


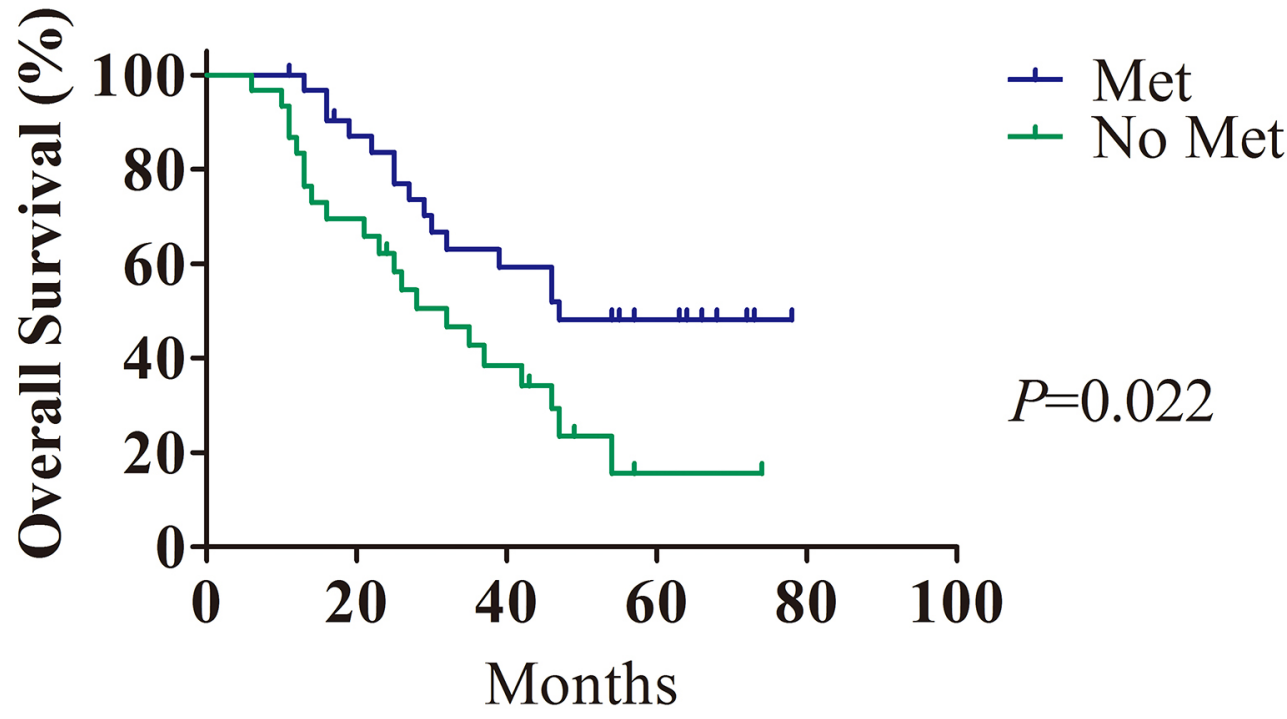

Figure 1: The prognostic value of metformin in HCC patients with T2DM. Treatment with metformin in HCC patients with T2DM ( $n=33$ ) was closely correlated with longer overall survival compared with treatment with other antidiabetic agents ( $n=30)$.

A

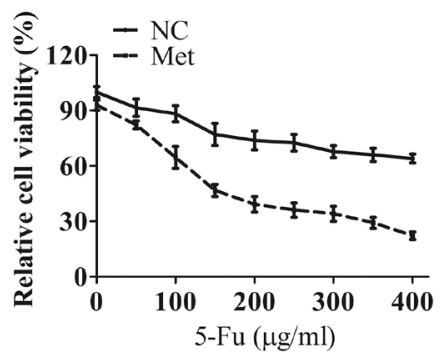

C

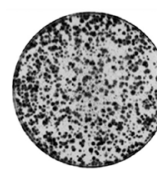

NC

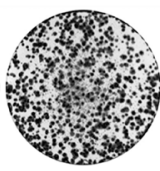

5-Fu

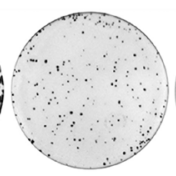

Met

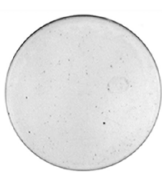

Com

B

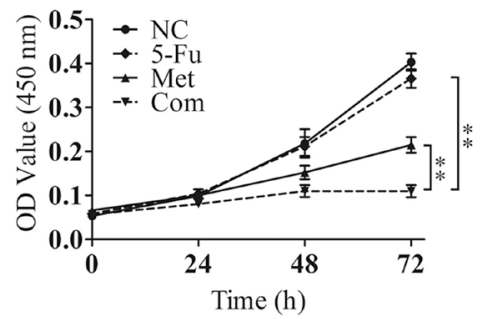

D

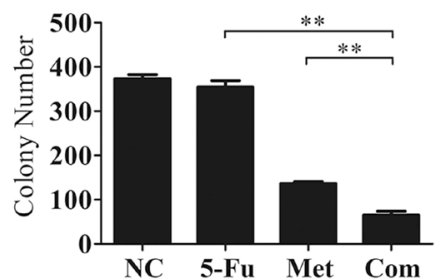

G

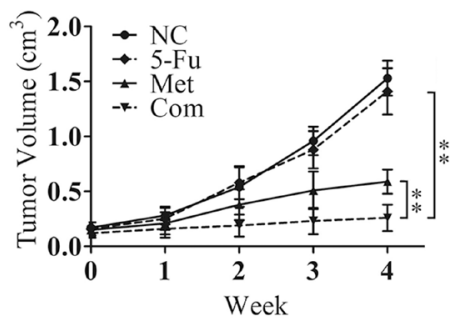

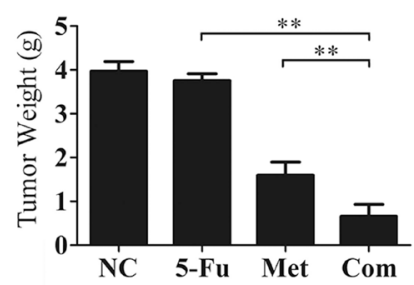

Figure 2: Metformin inhibits the proliferative capacity of Bel/Fu cells in combination with 5-Fu. A. The sensitivity of Bel/ Fu cells to 5-Fu was measured by CCK-8 assay. B. Bel/Fu cells were treated with different agents, and relative cell viability was measured by CCK-8 assay at 0 h, $24 \mathrm{~h}, 48 \mathrm{~h}$ and $72 \mathrm{~h}$. C and D. Bel/Fu cells were plated in $6 \mathrm{~cm}$ dishes at a density of 1000 cells per dish. After 2 weeks of different treatments, the number of colonies was counted. E. Combined treatment with 5-Fu and metformin decreased tumor size. The representative images of $\mathrm{Bel} / \mathrm{Fu}$ xenograft tumors injected subcutaneously and treated with NC, 5-Fu, Met and Com. F. The growth curve of tumors formed by Bel/Fu cells after treatment with $\mathrm{NC}, 5-\mathrm{Fu}$, Met and $\mathrm{Com}$. G. The weights of tumors formed by Bel/Fu cells after different treatments at harvest time. $* * \mathrm{P}<0.01$. 


\section{Silencing YAP mediates P-gp and MRP1 downregulation by inhibiting the phosphorylation of Akt}

Activation of Akt has been described in a variety of cancers, and the PTEN/Akt pathway plays a critical role in controlling the balance between pro- and antioncogenic pathways [21]. The aberrant expression of these proteins is always observed in cells that are not sensitive to chemotherapeutic agents [22]. Therefore, we examined the influence of YAP knockdown on PTEN, p-Akt and Akt protein levels by western blotting. As shown in Figure 5A, compared with the control cells, the knockdown of YAP in the Bel/Fu cells resulted in significant increases in PTEN, whereas the expression of p-Akt decreased remarkably but did not influence total Akt levels.

Hypoxia-inducible factor- $1 \alpha$ (HIF-1 $\alpha$ ) is a downstream protein of Akt that transcriptionally regulates

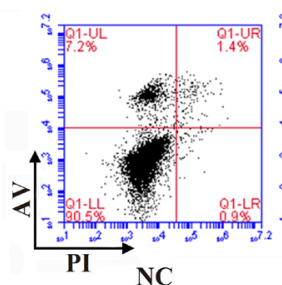

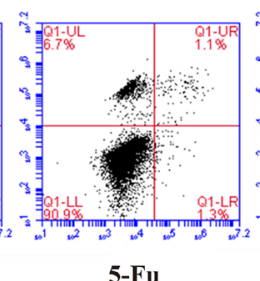

5-Fu

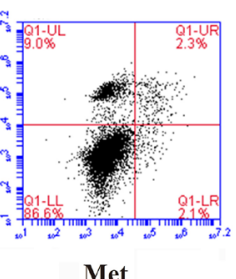

Met
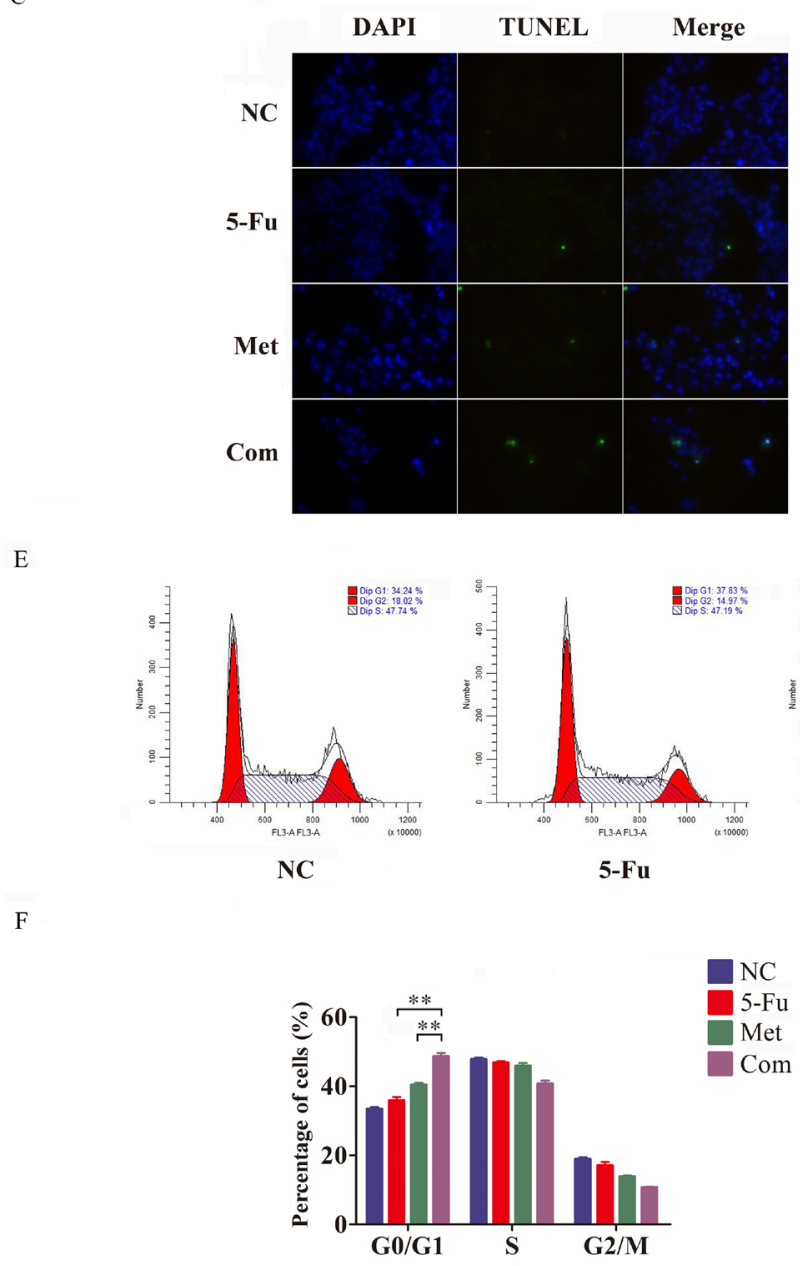

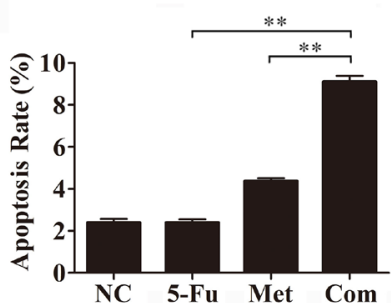

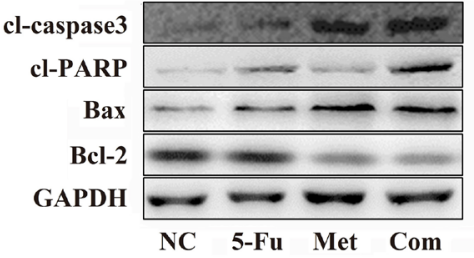

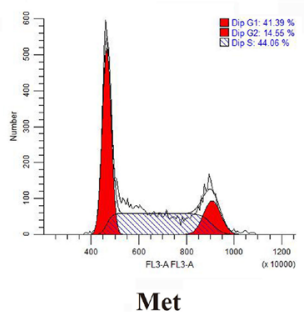

G
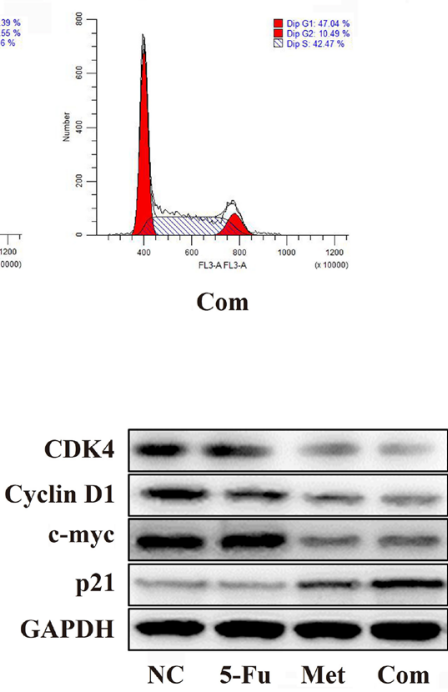

Figure 3: Metformin promotes apoptosis and arrests cell cycle progression in combination with 5-Fu in Bel/Fu cells. A and B. Bel/Fu cells were pretreated NC, 5-Fu, Met and Com separately. After $48 \mathrm{~h}$ of treatment, flow cytometry was performed to analyze the rate of cell apoptosis. C. TUNEL assay was used to detect DNA damage after the different treatments. D. The protein levels of cl-caspase3, cl-PARP, Bax, and Bcl-2 were detected by western blotting. $\mathbf{E}$ and $\mathbf{F} .24 \mathrm{~h}$ after different treatments, flow cytometry was performed to analyze the progression of the cell cycle. G. The protein levels of CDK4, cyclin D1, c-myc and p21 were detected by western blotting. $* * \mathrm{P}<0.01$. 
the expression of (P-glycoprotein) $\mathrm{P}$-gp and multidrug resistance-associated protein $1, \mathrm{MRP} 1$, which contribute to the development of chemoresistance [23]. Therefore, we investigated whether YAP could influence the expression of HIF-1 $\alpha$, P-gp and MRP1. Interestingly, western blotting illustrated that reduced expression levels of HIF-1 $\alpha, \mathrm{P}$-gp and MRP1 were detected in YAP siRNA\#2-transfected cells compared with control siRNA-transfected cells (Figure 5B). Therefore, these data may indicate a potential mechanism by which YAP regulates sensitivity to $5-\mathrm{Fu}$ in $\mathrm{Bel} / \mathrm{Fu}$ cells.

\section{Metformin downregulated expression of YAP and promoted YAP by activating AMPK}

It has recently been reported that AMPK activation can induce the phosphorylation of YAP, which is important in regulating cytokine transcription in the nucleus [24]. In our previous work, we confirmed that metformin is an AMPK activator. We further evaluated whether metformin could influence the phosphorylation of YAP. As expected, increased phosphorylation of YAP was observed in the Met and Com groups compared
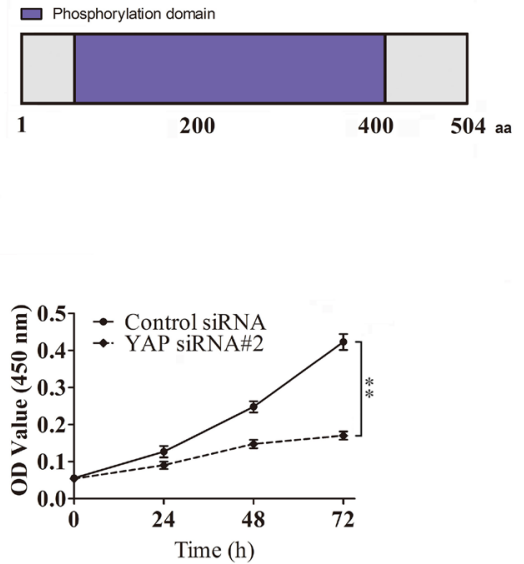

G

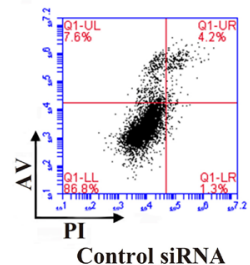

Control siRNA
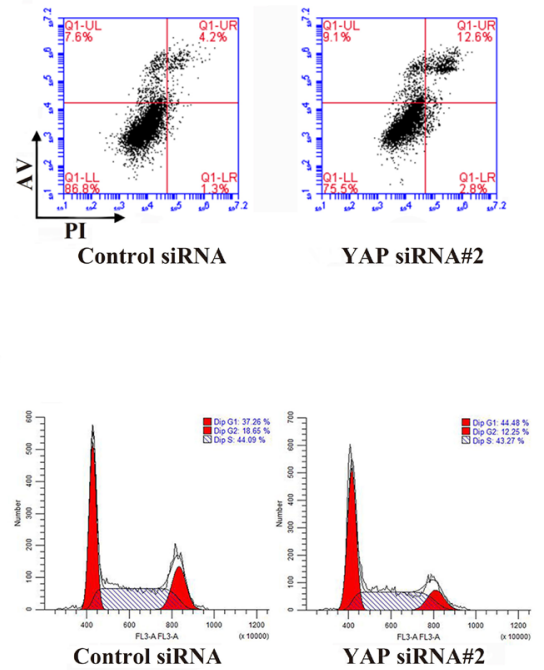

YAP siRNA\#2
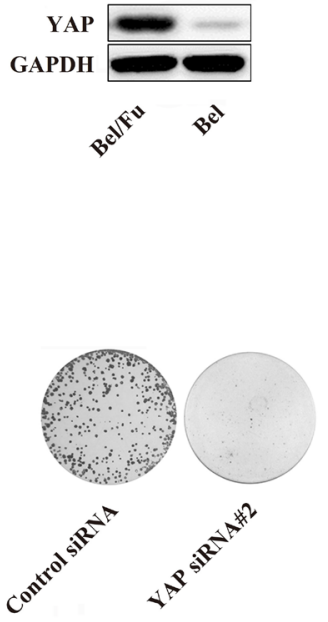

$\mathrm{H}$

K
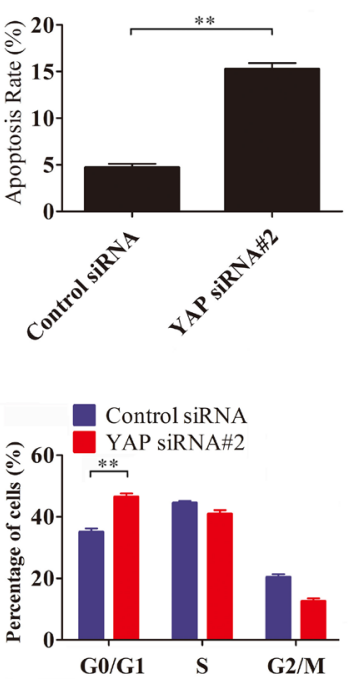
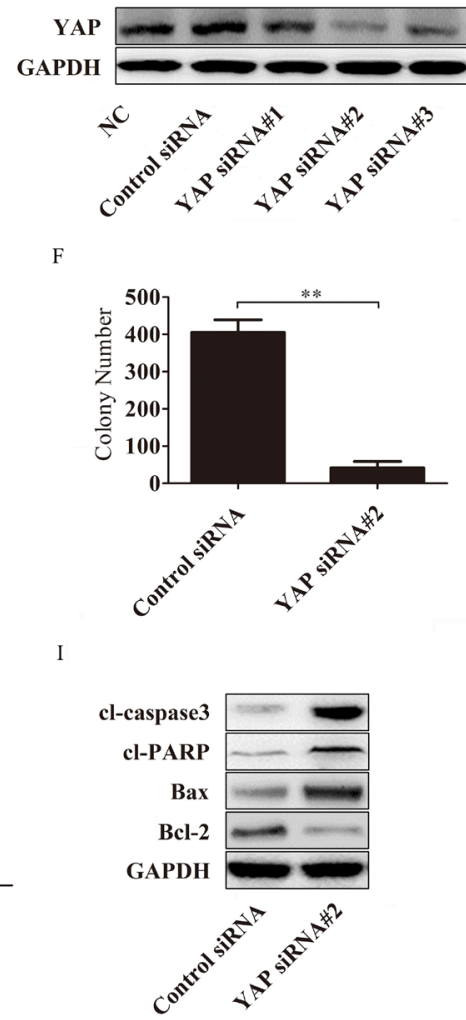

L

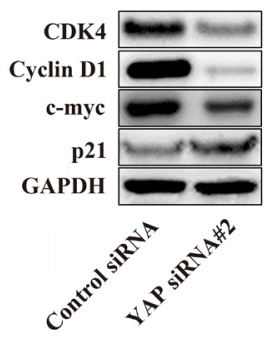

Figure 4: YAP knockdown resensitizes Bel/Fu cells to 5-Fu in Bel/Fu cells. A. A schematic illustration of the human YAP protein. YAP consists of 504 amino acid residues and has 22 phosphorylation sites. B. The expression of YAP in Bel/Fu and Bel cells. C. $\mathrm{Bel} / \mathrm{Fu}$ cells were transfected with siRNAs, and the expression of YAP was detected by western blotting. D. After the cells were transfected with control siRNA and YAP siRNA\#2, relative cell viability was measured by CCK-8 at different time points. $\mathbf{E}$ and $\mathbf{F}$. After being transfected with control siRNA and YAP siRNA\#2, the cells were cultured in $100 \mu \mathrm{g} / \mathrm{ml} 5$-Fu medium for 2 weeks, and colony numbers were counted. G and $\mathbf{H}$. Flow cytometry detected the rate of apoptosis in different siRNA-transfected cells. I. Detection of apoptosis-related proteins. $\mathbf{J}$ and $\mathbf{K}$. Flow cytometry detected cell cycle progression in different siRNA-transfected cells $24 \mathrm{~h}$ after siRNA transfection. L. Detection of cell cycle checkpoint proteins. $* * \mathrm{P}<0.01$. 
with the 5-Fu and the NC group, in agreement with the level of phosphorylation of AMPK. Moreover, we also discovered that the expression of YAP also decreased with metformin treatment (Figure 6A). These results indicated that activation of AMPK by metformin may suppress YAP with respect to the levels of total protein and phosphorylated protein.

\section{Metformin inhibited P-gp and MRP1 via the suppression of YAP}

In our study, we found that, in agreement with the previous presentation, metformin and combined treatment induced the phosphorylation of AMPK via the suppression of YAP. PTEN was significantly upregulated in the Met group and Com group, followed by the decreased phosphorylation of Akt (Figure 6B). Compared with the $\mathrm{NC}$ group and the 5-Fu group, the expression of HIF-1 $\alpha$, P-gp and MRP1 was obviously repressed (Figure 6C).

To verify whether YAP is a key regulator in metformin induced resensitivity to $5-\mathrm{Fu}$ in $\mathrm{Bel} / \mathrm{Fu}$ cells. We established YAP stable expression Bel/Fu cells. Western blotting showed YAP was overexpressed in Bel/ Fu-YAP than Bel/Fu-vector (Figure 6D). After treatment with $10 \mathrm{mM}$ metformin, Bel/Fu-YAP showed a lower expression of PTEN, and higher expression of $\mathrm{p}$-Akt than $\mathrm{Bel} / \mathrm{Fu}$-vector (Figure 6E). In addition, HIF-1 $\alpha, \mathrm{P}-\mathrm{gp}$ and MRP1 expressed higher in Bel/Fu-YAP cells (Figure $6 \mathrm{~F})$. CCK-8 analysis showed overexpression of YAP blocked metformin induced resensitivity to 5-Fu (Figure $6 \mathrm{G})$. These results further demonstrated that metformin may enhance chemotherapeutic sensitivity through the suppression of YAP.

A

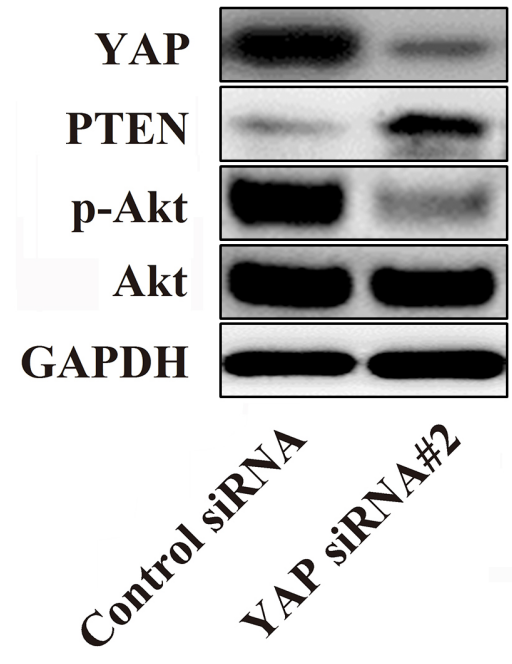

\section{DISCUSSION}

The low chemosensitivity of cancer cells has been implicated as a major challenge in anti-cancer treatment. Many factors have been identified in the development of insensitivity to chemotherapeutic agents: elevated expression of drug efflux transporters, changes in drug kinetics, amplification of drug targets or tumor heterogeneity, which comprise genetic variation, and the tumor microenvironment [25]. Ineffectiveness of chemotherapeutic agents in patients with advanced HCC has garnered the attention of increasing numbers of researchers.

Metformin, a biguanide derivative that is used widely to treat T2DM, reduces basal glucose output by suppressing gluconeogenesis and glycogenolysis in the liver and increasing glucose uptake by muscle. The mechanism involved in the effect of metformin is the liver kinase B1 (LKB1)-dependent activation of AMPK $[26,27]$. Many reports have demonstrated that there is an association between T2DM and various types of cancer [28]. In addition, the combination of metformin with conventional chemotherapeutic agents could also enhance chemosensitivity [29, 30], with little research focusing on HCC. In this study, we evaluated the efficiency of metformin and other antidiabetic agents in TACE treated HCC patients with T2DM. Similar to the findings of previous studies, metformin administration was associated with longer overall survival.

Although much progress has been made regarding the role of the Hippo pathway in tumorigenesis and stem cell renewal and differentiation $[12,31]$, its function with respect to the response to chemotherapeutic agents

$\mathrm{B}$

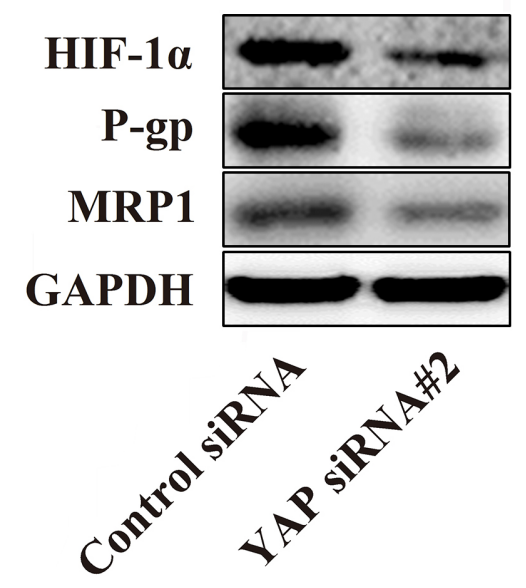

Figure 5: YAP regulates $P$-gp and MRP1 by regulating the phosphorylation of Akt in Bel/Fu cells. A. The knockdown of YAP increased the expression of PTEN and decreased the phosphorylation of Akt in Bel/Fu cells. B. The suppression of HIF-1 $\alpha$, P-gp and MRP1 was observed after YAP knockdown. 
is largely unknown. YAP, one of the most critical downstream effectors of the Hippo pathway, has been reported to form a complex with sequence-specific DNA-binding proteins and promotes the expression of genes that drive cell proliferation and inhibit apoptosis $[15,17]$. Phosphorylation of YAP deactivated YAP and translocated it from the nucleus to the cytoplasm, which abolished its transcriptional activity. Overexpression of YAP is associated with chemoresistance in esophageal cancer, and its interaction with the TEAD binding site in the EGFR promoter resulted in increased EGFR expression at the transcriptional level, causing resistance to 5-Fu and docetaxel [32]. Dephosphorylation of YAP at Ser 127 dissociated it from 14-3-3 binding, leading to its nuclear assembly and transcriptional activation [33]. To explore the biological function of YAP in HCC cells, YAP-specific siRNA was transfected into Bel/Fu cells. YAP knockdown suppressed cell growth, promoted cell apoptosis and induced G0/G1 cell cycle arrest in Bel/Fu cells treated with $5-\mathrm{Fu}$. Interestingly, Bel/Fu cells treated with metformin also showed suppression of YAP, which demonstrated that YAP is a crucial molecule in metformin induced 5-Fu resensitivity.

AMPK, a cellular energy stress sensor activated by increasing AMP levels, can modulate cellular metabolism and function to coordinate cell growth with available energy [34]. It has been reported that AMPK can inhibit YAP in a Lats-dependent and Latsindependent manner. AMPK phosphorylates and stabilizes angiomotin (AMOTL1) to stimulate Lats activity, thus phosphorylating YAP on five serine residues, including Ser 127 [35]. AMPK mediates the YAP phosphorylation, especially on Ser 94, plays a critical role in YAP inhibition by disrupting the formation of the YAP-TEAD complex [36]. Consistent with previous studies, metformin induced AMPK activation, phosphorylated YAP and decreased the expression of YAP, leading to the further inhibition of YAP in $\mathrm{Bel} / \mathrm{Fu}$ cells.

HIF-1 $\alpha$ has been recognized as a mediator of the development of chemoresistance. As the key stimulator of
A

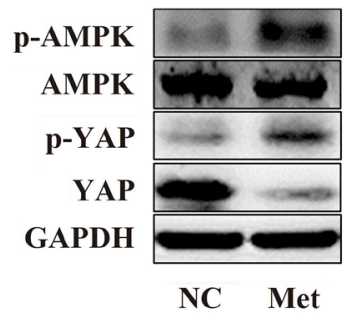

$\mathrm{D}$

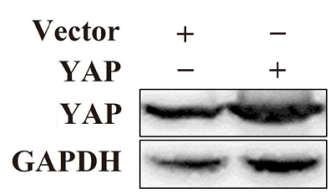

B

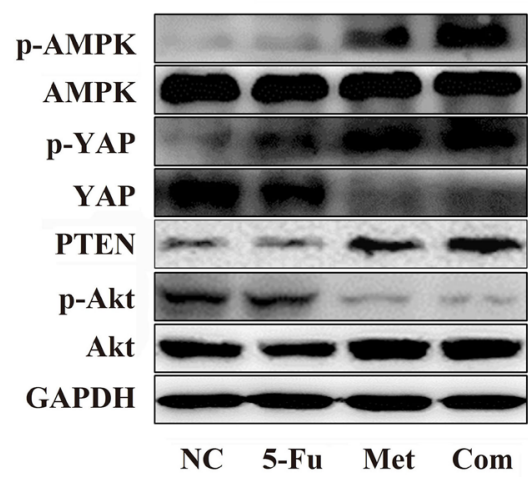

F

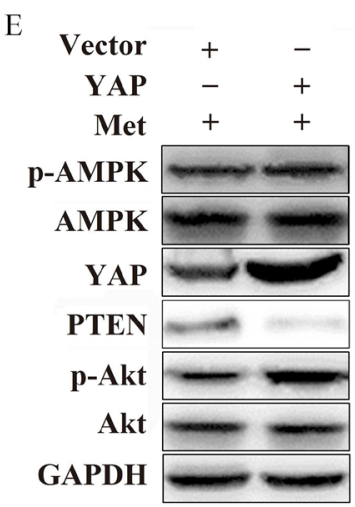

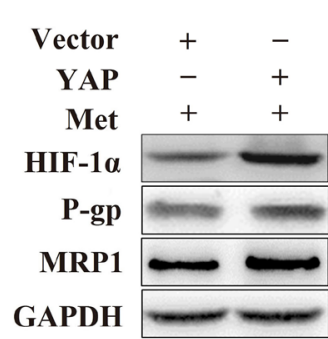

$\mathrm{C}$

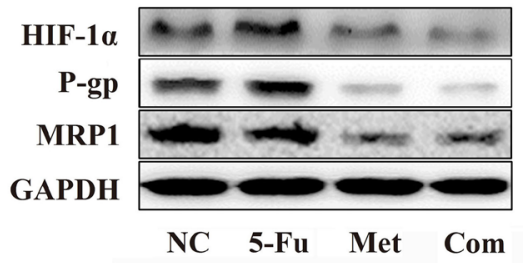

G

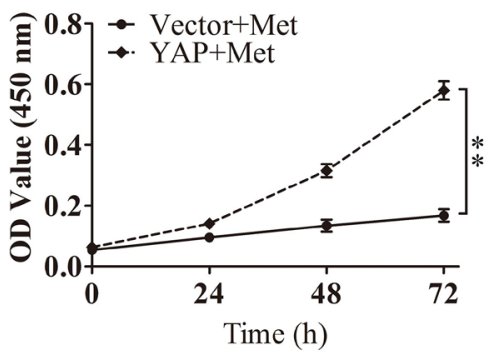

Figure 6: Metformin inhibits P-gp and MRP1 by suppressing YAP. A. Bel/Fu cells pretreated with metformin activated AMPK, decreased the expression of YAP, and inhibited the phosphorylation of YAP. B. Bel/Fu cells were treated with NC, 5-Fu, Met, and the Com, both PTEN protein levels and the levels of phosphorylated AMPK, YAP and Akt were examined using western blotting. C. HIF-1 $\alpha$, P-gp and MRP1 decreased in the Met group and the Com group compared with the NC group and the 5-Fu group. D. The expression of YAP in Bel/Fu-vector and Bel/Fu-YAP cells. E. After treated with metformin, Bel/Fu-YAP cells showed lower expression of PTEN and higher expression of p-Akt than Bel/Fu-vector cells. F. After treated with metformin, Bel/Fu-YAP cells showed higher expression of HIF-1 $\alpha$, P-gp and MRP1 than Bel/Fu-vector cells. G. CCK-8 assay for proliferation analysis between Bel/Fu-vector and Bel/Fu-YAP cells after metformin treatment. $* * \mathrm{P}<0.01$. 
HIF-1 $\alpha$, oxygen deprivation led to DDP and doxorubicin resistance in lung cancer cells [37]. P-gp and MDR1 are members of the drug transporter family, which are energydependent drug efflux pumps important in drug resistance [38]. They can be regulated by diverse signaling pathways, including the PI3K/Akt/HIF-1 $\alpha$ pathway [39]. Kunliang Guan et al provided evidences that YAP mediates the major effects of the Hippo pathway by regulating gene expression, and among the YAP target genes is the miR29 family, which inhibits PTEN, a tumor suppressor that inhibits the phosphorylation of Akt by targeting its 3'UTR [40]. In the current work, we silenced YAP and treated $\mathrm{Bel} / \mathrm{Fu}$ cells with metformin. Both managements led to the upregulation of PTEN, following downstream HIF$1 \alpha$ downregulation, and reduced the expression of P-gp and MRP1. Moreover, overexpression of YAP suprressed Akt activation, and blocked inhibition of P-gp and MRP1 induced by metformin. Therefore, we suggest that the downregulation of P-gp and MRP1 by metformin in Bel/ $\mathrm{Fu}$ cells may be caused by the suppression of YAP/PTEN/ Akt (Figure 7).

In conclusion, our findings revealed that $\mathrm{HCC}$ patients suffering from T2DM benefited from metformin administration, and the combination with metformin enhanced the sensitivity of Bel/Fu cells to 5-Fu both in vitro and in vivo. This study illustrates a novel mechanism by which metformin can be used as a prospective chemotherapeutic agent or chemosensitizer in $\mathrm{HCC}$ treatment.

\section{MATERIALS AND METHODS}

\section{Patients}

A total of $63 \mathrm{HCC}$ patients with type 2 diabetes mellitus (T2DM) who were diagnosed between March 2009 and December 2015 at the Second Affiliated Hospital of Dalian Medical University were retrospectively analyzed. All HCC patients received TACE treatment. The present study was approved by the Ethics Committees of the Second Affiliated Hospital of Dalian Medical University in accordance with the ethical guidelines of the Declaration of Helsinki. Consent was obtained from either the patient or the patient's family.

\section{Reagents and antibodies}

Metformin and 5-Fu were obtained from SigmaAldrich (St. Louis, MO, USA). The Cell Cycle Detection Kit, Cell Counting Kit-8, Annexin V-fluorescein isothiocyanate (FITC) Apoptosis Detection Kit, TUNEL Apoptosis Detection Kit and BCA protein assay kit were purchased from KeyGen Biotech Co., Ltd (Nanjing, China).

Antibodies against caspase3, cleaved-caspase 3 (clcaspase3), PARP, cleaved-PARP (cl-PARP), Bax, Bcl-2, p-YAP, YAP, p-AMPK, AMPK, Akt, p-Akt, PTEN, HIF1a, p21, c-myc and GAPDH were obtained from Santa Cruz Biotechnology, Inc. (Santa Cruz, CA, USA), the

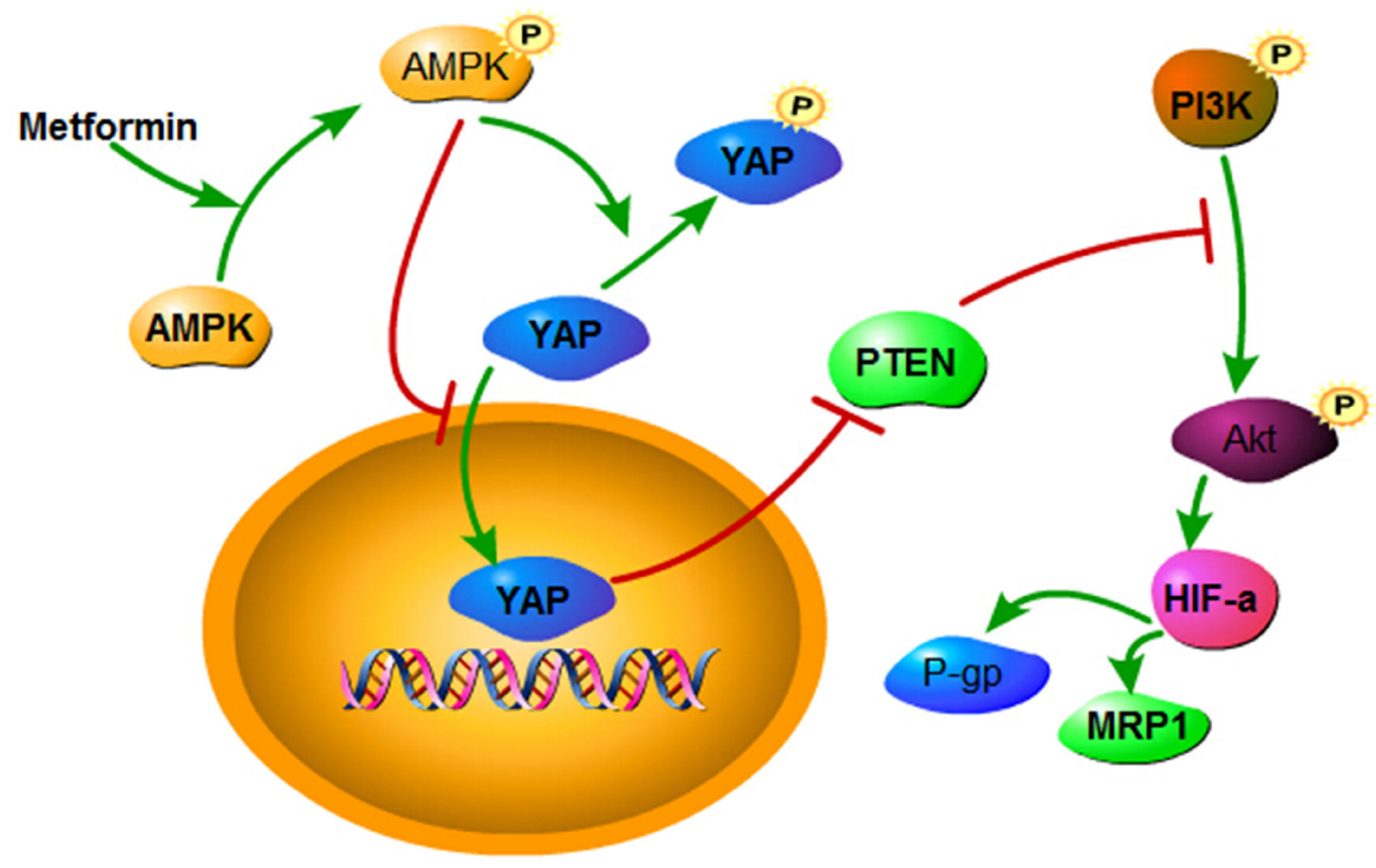

Figure 7: A schematic model elucidating the role of metformin in regulating the chemosensitivity of Bel/Fu cells. 
P-gp and MRP1 antibodies were obtained from Thermo Fisher Scientific Inc. (Rockford, IL, USA), and the cyclin D1 antibody was purchased from Abgent (San Diego, CA, USA).

\section{Cell culture and transfection}

The human hepatocellular carcinoma cell lines, Bel7402 (Bel) and Bel-7402/5-fluorouracil (Bel/Fu), were purchased from KeyGen Biotech Co., Ltd. HepG2, SKHep-1 were obtained from obtained from the tumor cell bank of the Chinese Academy of Medical Science. The cells were cultured in RPMI-1640 medium (Gibco-BRL, Carlsbad, CA, USA) supplemented with 10\% FBS (GibcoBRL, Carlsbad, CA, USA), $100 \mu \mathrm{g} / \mathrm{ml}$ penicillin and 100 $\mu \mathrm{g} / \mathrm{ml}$ streptomycin at $37^{\circ} \mathrm{C}$ in a $5 \% \mathrm{CO}_{2}$ atmosphere. The $\mathrm{Bel} / \mathrm{Fu}$ cells were maintained in medium containing 20 $\mu \mathrm{g} / \mathrm{ml} 5$-Fu. YAP specific siRNAs and full-length YAP complementary DNA were prepared and transfected as described previously $[19,41]$.

\section{Cell viability assay}

Approximately $5 \times 10^{3}$ cells/well were seeded into the wells of 96-well plates. After being cultured for 24 $\mathrm{h}$, the cells were treated with different agents or siRNA transfection. The cells were incubated at $37^{\circ} \mathrm{C}$ for another 24,48 or $72 \mathrm{~h}$, followed by exchanging the medium containing $100 \mu \mathrm{l}$ of RPMI-1640 medium and $10 \mu \mathrm{l}$ of CCK-8 reagent for $2 \mathrm{~h}$ at $37^{\circ} \mathrm{C}$. Finally, light absorbance was measured at $450 \mathrm{~nm}$ using a microplate reader.

\section{Colony formation assay}

Approximately 1000 cells/well were seeded in triplicate into 6-well plates. Then, $24 \mathrm{~h}$ after seeding, the cells were treated with different agents or siRNA transfection. After 2 weeks of growth, the cells were fixed and stained with crystal violet, and visible colonies were counted according to the cell numbers in each colony.

\section{In vivo tumor growth}

Nude mice were purchased from the SPF Laboratory Animal Center at Dalian Medical University. All animals were used in accordance with institutional guidelines, and the current experiments were approved by the Animal Care and Use Committee. Bel/Fu cells $\left(1 \times 10^{7} / 100 \mu \mathrm{l}\right)$ were resuspended in PBS and inoculated subcutaneously into 20 6-week-old nude mice. Two weeks later, when tumor diameters reached $4 \mathrm{~mm}$ to $5 \mathrm{~mm}$, the mice were randomly divided into 4 groups (n=5/group): $30 \mathrm{mg} / \mathrm{kg}$ 5 -Fu for the $5-\mathrm{Fu}$ group $(5-\mathrm{Fu}), 2 \mathrm{mg} / \mathrm{kg}$ metformin for the metformin group (Met), $30 \mathrm{mg} / \mathrm{kg} 5-\mathrm{Fu}$ and $2 \mathrm{mg} / \mathrm{kg}$ metformin for the combination treatment group (Com), and PBS alone for the control group (NC). These agents were intraperitoneally injected three times per week for six weeks. The tumors were measured weekly, and tumor volume was calculated according to the formula length $\times$ width $^{2} / 2$. The mice were terminated six weeks after inoculation, and the weights of the tumors in each mouse were measured.

\section{Flow cytometric analysis}

Cells were seeded into 6 -well plates $\left(2 \times 10^{5}\right.$ cells/ well). For cell cycle analysis, the cells were harvested and resuspended in $2 \mathrm{ml}$ of ice-cold $70 \%$ ethanol at $4{ }^{\circ} \mathrm{C}$ overnight. The fixed cells were centrifuged and washed with PBS. After incubation with $100 \mu \mathrm{l}$ of RNase A (10 $\mu \mathrm{g} / \mathrm{ml}$ ) for $30 \mathrm{~min}$ at $37^{\circ} \mathrm{C}$, the cells were resuspended in $400 \mu \mathrm{l}$ of PI $(50 \mu \mathrm{g} / \mathrm{ml})$ and placed in the dark at room temperature for $30 \mathrm{~min}$. For apoptosis analysis, the cells were digested and resuspended in binding buffer to prepare single cell suspensions. Then, the cells were stained using the Annexin V-FITC reaction reagent (5 $\mu \mathrm{l}$ of Annexin V-FITC, $5 \mu \mathrm{l}$ of propidium iodide) and incubated in the dark at room temperature for $30 \mathrm{~min}$. The stained cells were analyzed using an Accuri C6 flow cytometer.

\section{TUNEL assay}

Evidence of DNA strand breaks was detected using the transferase-mediated dUTP nick-end labeling (TUNEL) assay. The cells were cultured on coverslips for at least $24 \mathrm{~h}$ for attachment and then incubated with various treatments at $37^{\circ} \mathrm{C}$ for $48 \mathrm{~h}$. TUNEL assay was conducted according to the manufacturer's instructions. Fluorescence images were photographed at $\times 200$ magnification under a fluorescence microscope.

\section{Western blotting}

The cells were lysed in RIPA buffer on ice supplemented with $1 \mathrm{mM}$ PMFS and $1 \mathrm{mM}$ phosphatase inhibitor cocktail to obtain total cellular protein. Protein concentrations were determined using a BCA protein assay kit. The protein samples $(30 \mu \mathrm{g})$ were mixed with loading buffer, separated by $6 \%$ or $10 \%$ SDS-PAGE and transferred onto PVDF membranes. The membranes were then blocked and incubated with the primary and secondary antibodies. Then, the bands were visualized by chemiluminescence.

\section{Statistical analysis}

SPSS 17.0 statistical software was used for statistical analysis. All experiments were repeated three times. Values were presented as the mean \pm standard deviation. Student's t-test, one-way ANOVA and $\chi^{2}$ analysis were performed to analyze variance. Survival analysis was conducted using the Kaplan-Meier method, and the comparison of survival curves between groups was 
done with the log-rank test. A $P$-value $<0.05$ represented statistical significance.

\section{ACKNOWLEDGMENTS}

This research was supported by This research was supported by the National Natural Science Foundation of China $(81272368,81360367$ and 81471755), the Natural Science Foundation of Guangxi (2014GXNSFBA118162), and the Guangxi Health Department Project (Z2013464).

\section{CONFLICTS OF INTEREST}

No potential conflicts of interest were disclosed by all authors.

\section{REFERENCES}

1. Jemal A, Bray F, Center MM, Ferlay J, Ward E, Forman D. Global cancer statistics. CA Cancer J Clin. 2011; 61:69-90.

2. Hong W, Guan KL. The YAP and TAZ transcription co-activators: key downstream effectors of the mammalian Hippo pathway. Seminars in cell \& developmental biology. 2012; 23:785-793.

3. Forner A, Llovet JM, Bruix J. Hepatocellular carcinoma. Lancet (London, England). 2012; 379:1245-1255.

4. Fong ZV, Tanabe KK. The clinical management of hepatocellular carcinoma in the United States, Europe, and Asia: a comprehensive and evidence-based comparison and review. Cancer. 2014; 120:2824-2838.

5. Llovet JM, Bruix J. Systematic review of randomized trials for unresectable hepatocellular carcinoma: Chemoembolization improves survival. Hepatology (Baltimore, Md). 2003; 37:429-442.

6. Camma C, Schepis F, Orlando A, Albanese M, Shahied L, Trevisani F, Andreone P, Craxi A, Cottone M. Transarterial chemoembolization for unresectable hepatocellular carcinoma: meta-analysis of randomized controlled trials. Radiology. 2002; 224:47-54.

7. Sciarra A, Ronot M, Di Tommaso L, Raschioni C, Castera L, Belghiti J, Bedossa P, Vilgrain V, Roncalli M, Paradis V. TRIP: a pathological score for transarterial chemoembolization resistance individualized prediction in hepatocellular carcinoma. Liver international. 2015; 35:2466-2473.

8. Nagaoki Y, Hyogo H, Aikata H, Tanaka M, Naeshiro N, Nakahara T, Honda Y, Miyaki D, Kawaoka T, Takaki S, Hiramatsu A, Waki K, Imamura M, Kawakami Y, Takahashi S, Chayama K. Recent trend of clinical features in patients with hepatocellular carcinoma. Hepatology research. 2012; 42:368-375.

9. Koh WP, Wang R, Jin A, Yu MC, Yuan JM. Diabetes mellitus and risk of hepatocellular carcinoma: findings from the Singapore Chinese Health Study. British journal of cancer. 2013; 108:1182-1188.

10. Morales DR, Morris AD. Metformin in cancer treatment and prevention. Annual review of medicine. 2015; 66:17-29.

11. Galli GG, Carrara M, Yuan WC, Valdes-Quezada C, Gurung B, Pepe-Mooney B, Zhang T, Geeven G, Gray NS, de Laat W, Calogero RA, Camargo FD. YAP Drives Growth by Controlling Transcriptional Pause Release from Dynamic Enhancers. Molecular cell. 2015; 60:328-337.

12. Sudol M. Yes-associated protein (YAP65) is a proline-rich phosphoprotein that binds to the $\mathrm{SH} 3$ domain of the Yes proto-oncogene product. Oncogene. 1994; 9:2145-2152.

13. Guo L, Teng L. YAP/TAZ for cancer therapy: opportunities and challenges (review). International journal of oncology. 2015; 46:1444-1452.

14. Harvey KF, Zhang X, Thomas DM. The Hippo pathway and human cancer. Nature reviews Cancer. 2013; 13:246-257.

15. Overholtzer M, Zhang J, Smolen GA, Muir B, Li W, Sgroi DC, Deng CX, Brugge JS, Haber DA. Transforming properties of YAP, a candidate oncogene on the chromosome 11q22 amplicon. Proceedings of the National Academy of Sciences of the United States of America. 2006; 103:12405-12410.

16. Muramatsu T, Imoto I, Matsui T, Kozaki K, Haruki S, Sudol M, Shimada Y, Tsuda H, Kawano T, Inazawa J. YAP is a candidate oncogene for esophageal squamous cell carcinoma. Carcinogenesis. 2011; 32:389-398.

17. Zender L, Spector MS, Xue W, Flemming P, CordonCardo C, Silke J, Fan ST, Luk JM, Wigler M, Hannon GJ, $\mathrm{Mu} \mathrm{D}$, Lucito R, Powers S, Lowe SW. Identification and validation of oncogenes in liver cancer using an integrative oncogenomic approach. Cell. 2006; 125:1253-1267.

18. Shao DD, Xue W, Krall EB, Bhutkar A, Piccioni F, Wang X, Schinzel AC, Sood S, Rosenbluh J, Kim JW, Zwang Y, Roberts TM, Root DE, Jacks T, Hahn WC. KRAS and YAP1 converge to regulate EMT and tumor survival. Cell. 2014; 158:171-184.

19. Huo X, Zhang Q, Liu AM, Tang C, Gong Y, Bian J, Luk JM, Xu Z, Chen J. Overexpression of Yes-associated protein confers doxorubicin resistance in hepatocellullar carcinoma. Oncology reports. 2013; 29:840-846.

20. Marti P, Stein C, Blumer T, Abraham Y, Dill MT, Pikiolek M, Orsini V, Jurisic G, Megel P, Makowska Z, Agarinis C, Tornillo L, Bouwmeester T, Ruffner H, Bauer A, Parker $\mathrm{CN}$, et al. YAP promotes proliferation, chemoresistance, and angiogenesis in human cholangiocarcinoma through TEAD transcription factors. Hepatology (Baltimore, Md). 2015; 62:1497-1510.

21. Steelman LS, Chappell WH, Abrams SL, Kempf RC, Long J, Laidler P, Mijatovic S, Maksimovic-Ivanic D, Stivala F, Mazzarino MC, Donia M, Fagone P, Malaponte G, Nicoletti F, Libra M, Milella M, et al. Roles of the Raf/MEK/ERK and $\mathrm{PI} 3 \mathrm{~K} / \mathrm{PTEN} / \mathrm{Akt} / \mathrm{mTOR}$ pathways in controlling growth 
and sensitivity to therapy-implications for cancer and aging. Aging. 2011; 3:192-222. doi: 10.18632/aging.100296.

22. Lim HJ, Crowe P, Yang JL. Current clinical regulation of $\mathrm{PI} 3 \mathrm{~K} / \mathrm{PTEN} / \mathrm{Akt} / \mathrm{mTOR}$ signalling in treatment of human cancer. Journal of cancer research and clinical oncology. 2015; 141:671-689.

23. Lo YL, Liu Y. Reversing multidrug resistance in Caco-2 by silencing MDR1, MRP1, MRP2, and BCL-2/BCL-xL using liposomal antisense oligonucleotides. PloS one. 2014; 9:e90180.

24. DeRan M, Yang J, Shen CH, Peters EC, Fitamant J, Chan P, Hsieh M, Zhu S, Asara JM, Zheng B, Bardeesy N, Liu $\mathrm{J}, \mathrm{Wu} \mathrm{X}$. Energy stress regulates hippo-YAP signaling involving AMPK-mediated regulation of angiomotin-like 1 protein. Cell reports. 2014; 9:495-503.

25. Saunders NA, Simpson F, Thompson EW, Hill MM, EndoMunoz L, Leggatt G, Minchin RF, Guminski A. Role of intratumoural heterogeneity in cancer drug resistance: molecular and clinical perspectives. EMBO molecular medicine. 2012; 4:675-684.

26. Shaw RJ, Lamia KA, Vasquez D, Koo SH, Bardeesy N, Depinho RA, Montminy M, Cantley LC. The kinase LKB1 mediates glucose homeostasis in liver and therapeutic effects of metformin. Science. 2005; 310:1642-1646.

27. Hawley SA, Ross FA, Chevtzoff C, Green KA, Evans A, Fogarty S, Towler MC, Brown LJ, Ogunbayo OA, Evans AM, Hardie DG. Use of cells expressing gamma subunit variants to identify diverse mechanisms of AMPK activation. Cell metabolism. 2010; 11:554-565.

28. Libby G, Donnelly LA, Donnan PT, Alessi DR, Morris $\mathrm{AD}$, Evans JM. New users of metformin are at low risk of incident cancer: a cohort study among people with type 2 diabetes. Diabetes care. 2009; 32:1620-1625.

29. Tan BX, Yao WX, Ge J, Peng XC, Du XB, Zhang R, Yao B, Xie K, Li LH, Dong H, Gao F, Zhao F, Hou JM, Su JM, Liu JY. Prognostic influence of metformin as first-line chemotherapy for advanced nonsmall cell lung cancer in patients with type 2 diabetes. Cancer. 2011; 117:5103-5111.

30. Chen H, Yao W, Chu Q, Han R, Wang Y, Sun J, Wang D, Wang Y, Cao M, He Y. Synergistic effects of metformin in combination with EGFR-TKI in the treatment of patients with advanced non-small cell lung cancer and type 2 diabetes. Cancer letters. 2015; 369:97-102.

31. Sudol M, Harvey KF. Modularity in the Hippo signaling pathway. Trends in biochemical sciences. 2010; 35:627-633.
32. Yoshikawa K, Noguchi K, Nakano Y, Yamamura M, Takaoka K, Hashimoto-Tamaoki T, Kishimoto H. The Hippo pathway transcriptional co-activator, YAP, confers resistance to cisplatin in human oral squamous cell carcinoma. International journal of oncology. 2015; 46:2364-2370.

33. Wang P, Bai Y, Song B, Wang Y, Liu D, Lai Y, Bi X, Yuan Z. PP1A-mediated dephosphorylation positively regulates YAP2 activity. PloS one. 2011; 6:e24288.

34. Hardie DG. AMP-activated protein kinase: an energy sensor that regulates all aspects of cell function. Genes \& development. 2011; 25:1895-1908.

35. Zhao B, Wei X, Li W, Udan RS, Yang Q, Kim J, Xie J, Ikenoue T, Yu J, Li L, Zheng P, Ye K, Chinnaiyan A, Halder G, Lai ZC, Guan KL. Inactivation of YAP oncoprotein by the Hippo pathway is involved in cell contact inhibition and tissue growth control. Genes \& development. 2007; 21:2747-2761.

36. Li Z, Zhao B, Wang P, Chen F, Dong Z, Yang H, Guan KL, $\mathrm{Xu}$ Y. Structural insights into the YAP and TEAD complex. Genes \& development. 2010; 24:235-240.

37. Song X, Liu X, Chi W, Liu Y, Wei L, Wang X, Yu J. Hypoxia-induced resistance to cisplatin and doxorubicin in non-small cell lung cancer is inhibited by silencing of HIF-1alpha gene. Cancer chemotherapy and pharmacology. 2006; 58:776-784.

38. Zhu MM, Tong JL, Xu Q, Nie F, Xu XT, Xiao SD, Ran $\mathrm{ZH}$. Increased JNK1 signaling pathway is responsible for ABCG2-mediated multidrug resistance in human colon cancer. PloS one. 2012; 7:e41763.

39. Touil Y, Zuliani T, Wolowczuk I, Kuranda K, Prochazkova J, Andrieux J, Le Roy H, Mortier L, Vandomme J, Jouy N, Masselot B, Segard P, Quesnel B, Formstecher P, Polakowska R. The PI3K/AKT signaling pathway controls the quiescence of the low-Rhodamine123-retention cell compartment enriched for melanoma stem cell activity. Stem cells (Dayton, Ohio). 2013; 31:641-651.

40. Tumaneng K, Schlegelmilch K, Russell RC, Yimlamai D, Basnet H, Mahadevan N, Fitamant J, Bardeesy N, Camargo FD, Guan KL. YAP mediates crosstalk between the Hippo and PI(3)K-TOR pathways by suppressing PTEN via miR29. Nature cell biology. 2012; 14:1322-1329.

41. Shen Z, Stanger BZ. YAP regulates S-phase entry in endothelial cells. PloS one. 2015; 10:e0117522. 